\title{
Fortalecimiento de la comprensión lectora mediante la propuesta de Teresa Fuentes Navarro
}

Andrea del Pilar Rivera Rodríguez* Liliana el Pilar Rozo Romero*** María Cristina Hernández Pérez ${ }^{* * * *}$

Artículo de Investigación

Fecha de Recepción: 15 septiembre 2017

Fecha de Aprobación: 2 abril 2018

\section{Resumen}

La investigación realizada, tuvo como objetivo general presentar una propuesta para fortalecer la comprensión lectora a nivel literal e inferencial en estudiantes de grado cuarto y quinto de sectores rurales y urbanos, de una institución educativa del municipio de Tinjacá (Boyacá). A partir de un enfoque mixto, se orientaron cinco fases en la investigación que se describen a tomando el modelo procedimental que incluyó diagnóstico, planificación, aplicación, evaluación y revisión. Finalmente, como parte de la triangulación y contraste, se aplicó la batería de comprensión lectora PROLEC-SE-R, que complementó el análisis de resultados desde el nivel literal e inferencial. Las conclusiones evidenciaron las dificultades a nivel inferencial en cuanto a la deducción de algo que no sea explícito en el texto.

Palabras clave: comprensión lectora, investigación-acción, competencias básicas académicas, literal e inferencial.
* Institución Educativa Mariano Ospina Pérez, Boyacá-Colombia andreariverarodriguez@ yahoo.es

* Institución Educativa Mariano Ospina Pérez, Boyacá-Colombia lilirozo1201@gmail.com *** Institución Educativa Mariano Ospina Pérez, Boyacá-Colombia kristinaher13@gmail.com

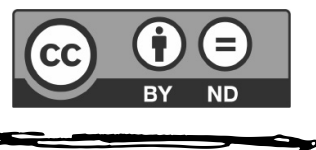




\section{Introducción}

Uno de los objetivos del Ministerio de Educación Nacional [MEN], consiste en promover programas y estrategias que permitan que los estudiantes colombianos logren un alto rendimiento académico, para desenvolverse de manera adecuada y productiva en las diversas áreas de su vida actual y futura.

Para lograrlo, desde las orientaciones epistemológicas, pedagógicas y curriculares definidas por el Ministerio de Educación Nacional [MEN], se han propuesto estrategias didácticas, orientadas por numerosos programas que se plantean desde las prácticas en el aula, las acciones institucionales, los convenios interinstitucionales y la formación permanente a los actores de los procesos educativos de nuestro país (MEN, 2006).

De allí, que la búsqueda permanente de la calidad educativa tenga el propósito de mejorar los aprendizajes, sobre todo en las áreas fundamentales del conocimiento, como es el lenguaje. Para analizar el impacto de las estrategias utilizadas por los docentes en las instituciones educativas tanto públicas como privadas del país, se contó con la valoración periódica que se evalúa a través de las pruebas internas y externas a nivel regional, nacional e internacional.

Cada vez resulta más difícil ignorar que la evaluación externa, las evaluaciones censales y muestrales aplicadas anualmente como son las pruebas SABER y el índice sintético de calidad, han tomado un valor determinante en el destino de las instituciones educativas, no solo por evaluar las destrezas, habilidades y valores independientemente de su procedencia, condiciones sociales, económicas y culturales, sino, por asociar los resultados con los desempeños de los estudiantes.

La investigación que se presenta a continuación, espera responder al interrogante ¿qué aportes generará la implementación de la propuesta de Teresa Fuentes Navarro, en el fortalecimiento de la comprensión lectora a nivel literal e inferencial en estudiantes del grado cuarto y quinto de la institución educativa Mariano Ospina Pérez?

De igual forma, los objetivos estuvieron encaminados a fortalecer los procesos de comprensión lectora a nivel literal e inferencial de niños estudiantes en básica primaria, de sectores rurales y urbanos, matriculados en los grados cuarto y quinto, respectivamente. Apoyados en el análisis individual de su desempeño, hasta la puesta en marcha de la propuesta de Fuentes (2010), se referenciaron los resultados obtenidos de la aplicación en una evaluación de triangulación y contraste, que a través del enfoque cualitativo, nos permitió comprender e interpretar el fenómeno de estudio desde una perspectiva más contextualizada, acercada a los escenarios, características y condiciones particulares de nuestros estudiantes de la institución educativa Mariano Ospina Pérez.

Los fundamentos teóricos que enriquecieron esta investigación, 
iniciaron desde el abordaje histórico de Rey (2000) principios del método Decroly, la conceptualización de la comprensión lectora desde la perspectiva de Pérez (2005), García (2008), los niveles de comprensión literal e inferencial referenciados por Méndez (2006).

Subsecuentemente Tobón (2001) y Cassany \& Sáenz (1994) han analizado la problemática producto del analfabetismo y las dificultades prácticas de enseñanza de la lengua y su compresión. De allí, la necesidad de abarcar un tema que compete al sistema educativo, los docentes, estudiantes, padres de familia y a la sociedad en general, llevó a los autores a describir los procesos necesarios en la comprensión lectora, los tipos de lectura, el perfil del lector, los modelos de comprensión lectora, las micro habilidades y la didáctica.

Apoyados en las estrategias de lectura de Solé (1992) se realizó un recuento teórico de la lectura, su comprensión y aprendizaje. A partir de sus postulados, adoptamos estrategias metodológicas en la aplicación de las cartillas de Teresa Fuentes. La propuesta de secuencia didáctica para la enseñanza de la comprensión lectora orientada por Solé, apoyó las actividades de la intervención educativa. Las herramientas necesarias para la puesta en marcha de los aspectos metodológicos de valoración, recibieron su apoyo en Ramos, Cuetos \& Rivas (2016), Pérez (1990), Elliot (1990), Fuentes (2007), Borjas (2006) y Hernández (2014). Finalmente, a través de estas reflexiones y otros tantos autores expertos en el tema, se analizaron las perspectivas de la educación basada en competencias, la evaluación y optimización educativa, la función de la evaluación de programas educativos y análisis del modelo educativo institucional.

\section{Materiales y métodos}

La población estuvo conformada por estudiantes de grado cuarto y quinto, matriculados en la institución educativa Mariano Ospina Pérez; niños y adolescentes en un rango de edad de 9 a 14 años, procedentes de familias ubicadas en la provincia de Ricaurte del Municipio de Tinjacá del Departamento de Boyacá, cuya muestra fue extraída a través del método no probabilístico. En la primera etapa, se seleccionaron 38 estudiantes matriculados en la sede centro en el grado cuarto (18) y quinto (20); en una segunda etapa, fueron escogidos estudiantes del sector rural matriculados en la sede Funza. Allí, de un total de 25 estudiantes, en los grados cuarto y quinto, se encontraron matriculados un total de 13 estudiantes; en la tercera etapa, se tuvo en cuenta toda la muestra poblacional a nivel rural y urbana, fueron categorizados estudiantes de la institución que no presentaran diagnósticos registrados en el sistema de matrícula SIMAT, en la categoría de necesidades educativas especiales $y / 0$ inclusión educativa. Uno de los estudiantes presentó dicha categoría, por esta razón, la muestra poblacional final total se constituyó en 50 estudiantes.

Los procedimientos nombrados, fueron seleccionados de acuerdo
Tobón (2001) y Cassany \& Sáenz (1994) han analizado la problemática producto del analfabetismo y las dificultades prácticas de enseñanza de la lengua y su compresión. 
En el proceso de observación, se realizó bajo el reconocimiento de 6 elementos sugeridos por Benguría (2010). El nivel planteado para el estudio fue no sistemático. con el planteamiento del problema; esto teniendo en cuenta, que, en las pruebas externas nacionales (SABER) e internacionales (PIRLS), solo participaron estudiantes sin categorías diagnósticas. Así mismo, para la presente investigación, la ventaja de la muestra no probabilística se plantea desde la postura de Sampieri (2010).

\section{La propuesta metodológica}

La investigación-acción educativa fue seleccionada por su carácter práctico, reflexivo, social, intencionalidad y por los aspectos procedimentales y metodológicos. Desde la perspectiva educativa, Suárez Pazos, citado en (Colmenares \& Piñero, 2008), refiere que la investigación-acción es "una forma de estudiar, de explorar, una situación social, en nuestro caso educativa, con la finalidad de mejorarla, en la que se implican como "indagadores" los implicados en la realidad investigada". (p. 34)

La presente investigación contempló las siguientes fases:

Fase inicial: Parte desde el "Diagnóstico: Identificación del problema, necesidades y recursos". Pérez (1990) incluyó: (a) Gestión institucional: permisos escritos ante directivos docentes para llevar a cabo la presente investigación; (b) Diseño del protocolo de consentimiento y aceptación: para la participación en el proyecto de investigación dirigido a padres y/o acudientes; de igual forma, la estructuración del asentimiento para estudiantes. Los dos protocolos fueron fundamentados en los principios éticos y la reglamentación de la Asociación Psicológica Americana (APA), tomados de Kantowitz, Elmes \& Roediger (2001); (c) Revisión de documentos institucionales: incluyó la lectura del Proyecto Educativo Institucional, antecedentes investigativos, consulta del índice sintético de calidad, resultantes de las pruebas internas y externas; (d) Implementación de la técnica de observación: desde la perspectiva de Borjas et al., (2006).

En el proceso de observación, se realizó bajo el reconocimiento de 6 elementos sugeridos por Benguría (2010). El nivel planteado para el estudio fue no sistemático. Las estrategias utilizadas en el proceso se realizaron bajo la modalidad de la observación participante, con la técnica directa de esta y la ocurrencia de campo. Posteriormente, se realizó la aplicación de la actividad diagnóstica propuesta del Ministerio de Educación Nacional [MEN]: consta de 23 preguntas para el grado cuarto y 27 preguntas para el grado quinto "que permiten identificar el desarrollo de los procesos de aprendizaje de los estudiantes de grado cuarto y quinto en el área de lenguaje, identificando los niveles de desempeño que permiten la generación de hipótesis sobre las posibles dificultades en la comprensión” (MEN, 2012).

Fase de acción: se plantea desde la "Planificación: objetivos generales y específicos, metodología estrategias y tareas, tiempos, ubicación y recursos" Pérez (1990). 
Se seleccionó la propuesta competencias básicas académicas (CAB) cuya autora es Teresa Fuentes, Eiras, et al. (2018). El programa está compuesto por cuatro cuadernillos, titulados: Manual de aplicación, cómo mejorar mis calificaciones, comprensión de lectura y manual para elegir fundamentalmente una ocupación.

Atendiendo a los objetivos de la investigación, se realizó la selección del cuadernillo de comprensión lectora. El objetivo de la cartilla consistió en "que los estudiantes aprendan y optimicen las competencias básicas de leer y comprender diversos textos" Fuentes (2010).

La cartilla de comprensión lectora está compuesta por cinco apartados: (a) distinción de elementos; (b) parafraseo; (c) análisis; (d) establecimiento de semejanzas y diferencias; y (e) síntesis. De conformidad con los objetivos de la investigación, fueron seleccionados tres apartados en las categorías de actividades con contenido literal e inferencial.

Fase de aplicación: Se planteó desde los aspectos del "desarrollo, seguimiento y control” Pérez (1990). El programa de competencias básicas académicas cuenta con un manual de aplicación con las sugerencias metodológicas. El desarrollo de la cartilla se realizó de forma sistemática y gradual.
Fase de evaluación: Se proyectó desde los aspectos de evaluación, diagnóstico y proceso final Pérez (1990). Se aplicó la batería de contraste PROLEC-SE -Evaluación de los procesos lectores- de Ramos, Cuetos \& Arrivas (2016), cuyo objetivo es la evaluación de los procesos lectores, en las categorías de capacidad lectora y los procesos cognitivos que intervienen en la lectura. Las tareas evaluadas incluyeron la evaluación de los procesos semánticos a nivel literal e inferencial.

\section{Resultados}

Los resultantes del proceso de observación destacaron las dificultades de los estudiantes, en aspectos como: escasos hábitos de lectura, inconvenientes en la comprensión de aspectos formales y conceptuales en el interior de cada texto leído, así como también para la identificación de los tipos de textos y su intención comunicativa.

La actividad diagnóstica propuesta por el Ministerio de Educación Nacional [MEN], de alguna forma, reflejó dificultades en los procesos de comprensión de diversos tipos de texto que implican la utilización de estrategias de búsqueda en el texto y la fundamentación pedagógica para lograrlo (Ver tabla 1). 
Tabla 1

Resultados Evaluación Diagnóstica

\begin{tabular}{ccccc}
\hline Nivel & $\begin{array}{c}\text { Grado cuarto } \\
\text { Sede Rural } \\
\text { Funza }\end{array}$ & $\begin{array}{c}\text { Grado quinto } \\
\text { Sede Rural } \\
\text { Funza }\end{array}$ & $\begin{array}{c}\text { Grado cuarto } \\
\text { Sede Centro }\end{array}$ & $\begin{array}{c}\text { Grado quinto } \\
\text { Sede Centro }\end{array}$ \\
\hline $\begin{array}{c}\text { Respuestas correctas } \\
\text { Nivel literal }\end{array}$ & $59,50 \%(\mathrm{n}=50)$ & $63,40 \%(\mathrm{n}=40) \mathrm{v}$ & $50,80 \%(\mathrm{n}=121)$ & $62,20 \%(\mathrm{n}=112)$ \\
\hline $\begin{array}{c}\text { Respuestas } \\
\text { incorrectas Nivel } \\
\text { literal }\end{array}$ & $40,50 \%(\mathrm{n}=34)$ & $36,60 \%(\mathrm{n}=23)$ & $49,20 \%(\mathrm{n}=117)$ & $37,80 \%(\mathrm{n}=68)$ \\
\hline $\begin{array}{c}\text { Respuestas correctas } \\
\text { nivel inferencial }\end{array}$ & $35,10 \%(\mathrm{n}=19)$ & $51 \%(\mathrm{n}=64)$ & $74,60 \%(\mathrm{n}=113)$ & $62,80 \%(\mathrm{n}=226)$ \\
\hline $\begin{array}{c}\text { Respuestas } \\
\text { incorrectas nivel } \\
\text { inferencial }\end{array}$ & $64,90 \%(\mathrm{n}=35)$ & $49 \%(\mathrm{n}=62)$ & $25,40 \%(\mathrm{n}=39)$ & $37,20 \%(\mathrm{n}=134)$ \\
\hline
\end{tabular}

Nota. Fuente: (Ministerio de Educación Nacional [MEN], 2006).

Los resultados reflejan que los estudiantes seocuparon delas ideas expuestas de modo explícito, identificando informaciones, tales como: personajes, acontecimientos, lugares donde se desarrollan las acciones y el tiempo cuando estas transcurren CR, MEP (2006).

Con un porcentaje mayoritario, los resultados evidencian que los estudiantes presentaron dificultad en la elaboración de interpretaciones, conjeturas e hipótesis, por parte del lector con base tanto en la información explícita e implícita como en sus propias intuiciones y experiencias personales lo que se traduce en mayores dificultades de tipo inferencial.

Respecto de la aplicación del tratamiento educativo, mediante la cartilla de comprensión lectora en los tres apartados, se pudo evidenciar que, en el apartado 1 (distinción de elementos), los estudiantes distinguieron los elementos del texto referentes a la especificación de los aspectos explícitos más importantes del mismo. En el apartado 3 (análisis), los estudiantes no solo analizaron la reconstrucción del texto, sino también el establecimiento de nuevas relaciones acerca o a partir de este. Finalmente, en el apartado 4 (diferencias y semejanzas), los estudiantes pudieron establecer diferencias y semejanzas entre los elementos del texto, que consistió en identificar los elementos comparables del texto de acuerdo con un criterio particular y en especificar aquello en lo que se asemejaba o difería.

Las dificultades porcentuales se dejaron ver en la resolución de los ejercicios propuestos por las cartillas en la categoría inferencial, tanto para la sede rural como para la urbana. Su análisis se presenta por cada grado de escolaridad y se evidencia por cada una de las instituciones educativas a fin de entregar unos resultados que fueron verificados, lo que dio a este estudio de investigación una alta validez y consistencia interna (ver tabla 2). 
Tabla 2

\section{Resultados Evaluación Cartilla Comprensión Lectora}

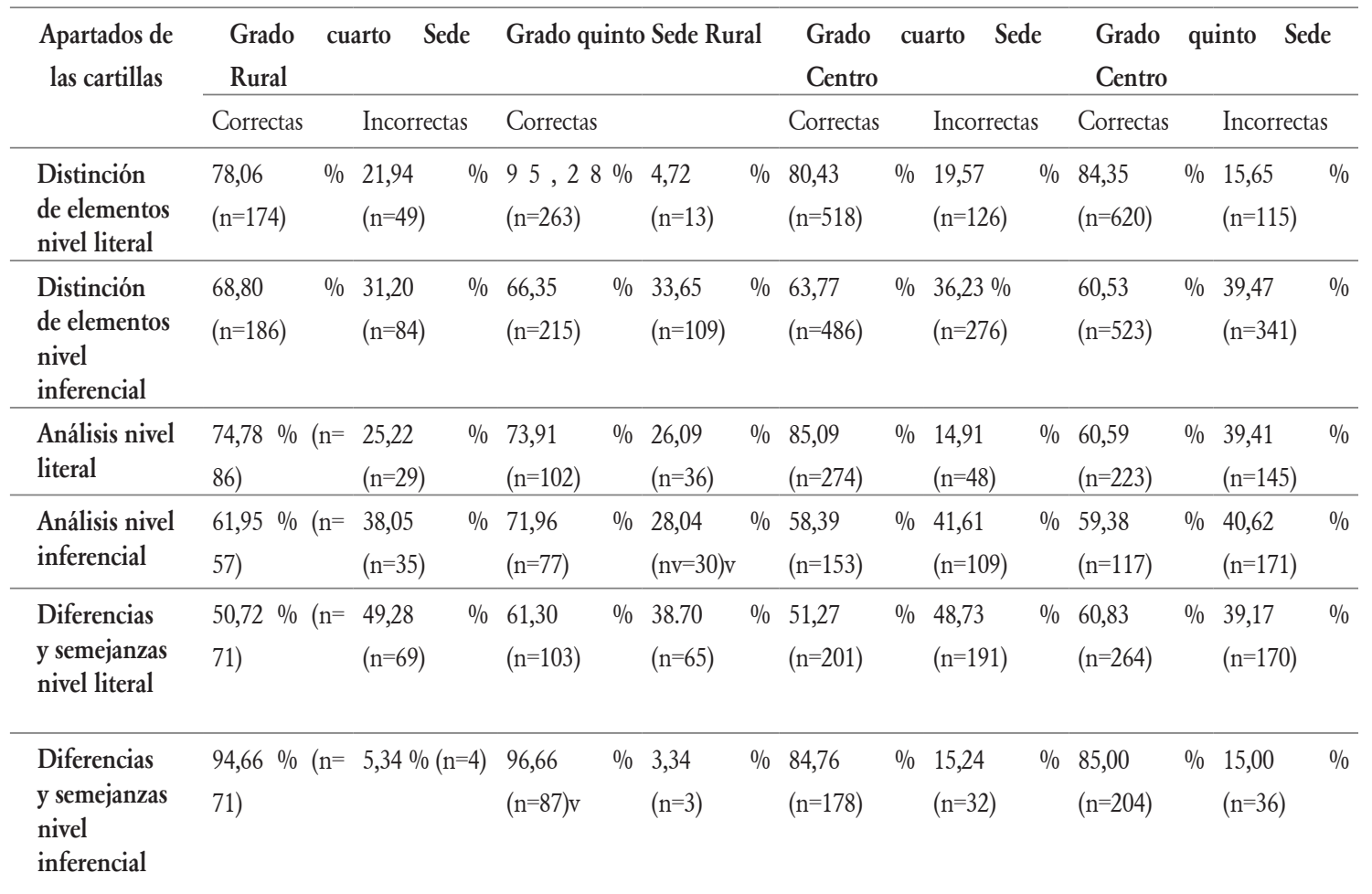

Fuente: (Fuentes , 2007)

Los resultados de la prueba PROLECSE, ubicó a los estudiantes de grado quinto en el nivel medio, lo que significa que la puntuación está entre la media y Dt por encima de la media. Su rendimiento es el de la mayoría de los estudiantes de su edad y curso.

El porcentaje mayoritario de los estudiantes se ubicó en el nivel Bajo, lo que significa que la puntuación directa está entre la media y 1 Dt por debajo de la media. Su rendimiento es normal, aunque ligeramente bajo, en comparación con otros estudiantes de su edad y curso. Para todos los estudiantes, la prueba puntuó un nivel de dificultad en la categoría NO, lo que significa que la puntuación directa de los estudiantes es superior a 1Dt por debajo de la media, lo que se traduce en la no presentación de dificultades en esta área (ver tabla 3). 
Tabla 3

Evaluación de los Procesos Lectores

\begin{tabular}{|c|c|c|c|c|c|c|c|c|}
\hline & \multicolumn{2}{|c|}{ Grado cuarto Sede Rural } & \multicolumn{2}{|c|}{ Grado quinto Sede Rural } & \multicolumn{2}{|c|}{ Grado cuarto Sede Centro } & \multicolumn{2}{|c|}{ Grado quinto Sede Centro } \\
\hline & NIVEL & DIFICULTAD & $\begin{array}{l}\text { N } \\
\text { I } \\
\text { VE } \\
\text { L }\end{array}$ & DIFICULTAD & NIVEL & DIFICULTAD & NIVEL & DIFICULTAD \\
\hline LITERAL & Bajo & No & Medio & No & Bajo & No & Bajo & No \\
\hline INFERENCIAL & Bajo & No & Medio & No & Bajo & No & Bajo & No \\
\hline
\end{tabular}

Fuente: Resultados batería PROLEC-SE

\section{Conclusiones}

Algunos factores de incidencia que se concluyen del proceso de observación, destacaron las dificultades de los estudiantes en aspectos como: escasos hábitos de lectura, inconvenientes en la comprensión de aspectos formales y conceptuales en el interior de cada texto leído, así como también para la identificación de los tipos de textos y su intención comunicativa.

La investigación realizada buscó logró contribuir con la institución educativa Mariano Ospina Pérez, en la medida en que aportó una triangulación de los aspectos cuantitativos y cualitativo.

En tal sentido Ramos, Cuetos \& Arrivas, (2016) precisaron:

"Respecto a la comprensión de texto, se comprobó que los alumnos tienen más dificultades en responder a las preguntas inferenciales que a las literales. Esto indica que, en muchos casos, no llegaron a realizar una comprensión total del texto que leen. Esta prueba junto con la de identificación de la estructura del texto son las que implica mayor complejidad cognitiva, por ello son las que mejor van a predecir la eficacia lectora"; de ésta manera estamos aproximándonos al análisis específico de la problemática en nuestro contexto. (pág. 18)

Los resultados son concluyentes respecto de: (a) la necesidad de evaluar de forma diagnóstica y contextualizada los procesos lectores; (b) la necesidad de aportar un tratamiento educativo coherente con las particularidades del contexto y situación académica; (c) la importancia de realizar un seguimiento a los procesos ya iniciados y revalorar los objetivos planteados inicialmente frente a cualquier proceso académico que implique la comprensión lectora y (d) el requisito indispensable que se debe tener en cuenta, y constituye la redacción que se pudo recolectar de los procesos aplicados que sirvieron como punto de partida en las estrategias planteadas en los planes de estudio, los planes de mejoramiento institucional; así como herramientas prácticas que están a disposición de ser usadas por los docentes en el proceso pedagógico y metodológico. 


\section{Referencias}

Benguria, M. (2010). Métodos de investigación en educación especial. Observación, 16-46. Recuperado el 23 de Marzo de 2019, de http://ayura.udea.edu.co:8080/ jspui/bitstream/123456789/2502/1/D0258_alejandra_iris_luisa.pdf

Borjas, P. (2006). Propuesta didactica para la enseñanza de la lectura y la escritura en la educación basica. (F. y. integral, Ed.) Maracaibo: Fe y Alegria. Recuperado el 22 de Marzo de 2019, de https://es.slideshare.net/marrisan/propuesta-didctica-para-laenseanza-de-la-lectura-y-la-escritura-en-la-educacin-bsica

Cassany, L., \& Saénz. (1994). Enseñar lengua. Ed. GRAÓ, de IRIF, S.L. C/ Francesc Tárrega.

Colmenares, M., \& Piñero, L. (2008). La investigación. Una herramienta metodológica heurística para la comprensión y transformación de realidades y prácticas socioeducativas. Laurus, 14(27), 96-114.

Elliot, J. (1990). La investigación-acción en educación (6a. ed.). Morata S.L. Recuperado el 21-03-2015 de Marzo de 2019, de http://www.edmorata.es/libros/la-investigacionaccion-en-educacion

Fuentes, M. (2007). Los conceptos académicos desde la perpectiva interconductual. Acta colombiana de psicología, 53-54.

Fuentes, M. (2010). Competencias Académicas Básicas. Buenos Aires, Argentina: Manual Moderno. Recuperado el 21 de Marzo de 2019, de http://cas.ides.org.ar/ files/2017/05/EAS_V1N2NS_08_INV_meri.pdf

García, M. (2008). Memoria operativa, comprensión lectora y razonamiento en la educación secundaria. Anuario de Psicología, 39(01), 133-157.

Hernández, S. (2014). Metodología de la investigación (6a. ed.). México: Ma Graw Hill. Recuperado el 22 de Marzo de 2019, de http://observatorio.epacartagena.gov. co/wp-content/uploads/2017/08/metodologia-de-la-investigacion-sexta-edicion. compressed.pdf

Cuetos, F., \& Arrivas, D. (2016). Evaluacion de los procesos lectores en secundaria y bachillerato PROLEC-SE-R. Mexico: TEA. Recuperado el 21 de Marzo de 2019, de respecto a la comprensión de texto, hemos comprobado que los alumnos tienen más dificultades en responder a las preguntas inferenciales que a las literales. Esto indica que, en muchos casos, no llegan a realizar una comprensión total del texto que leen. $\mathrm{E}$

Kantowitz, R., Elmes, D., \& Roediger, H. (2001). Psicologia experimental. S.A. Ediciones paraninfo. Recuperado el 22 de Marzo de 2019, de https://atam.casadellibro. com/libro-psicologia-experimental-7-ed/9789706860729/793529

Mendez, S. (2006). Comprensión lectora y textos literarios: una propuesta psicopedagógica. Educación, 30(01), 141-155. 
Ministerio de Educación Nacional [MEN]. (Mayo de 2006). Estándares básicos de competencias en lenguaje, matemáticas, ciencias y ciudadanas. (M. d. Nacional, Ed.) Revolución Educativa, Colombia aprende(01), 14-15. Recuperado el 21 de Marzo de 2019, de https://www.mineducacion.gov.co/1621/articles-116042_ archivo_pdf.pdf

Ministerio de Educación Nacional. (2006). Estándares básicos de competencias en lenguaje, matemáticas y ciencias ciudadanas. Bogotá: Ministerio de Educación Nacional. Recuperado el 22 de Marzo de 2018, de https://www.mineducacion.gov.co/1621/ articles-116042_archivo_pdf.pdf

Ministerio de Educación Nacional, MEN. (2012). Estándares básicos de competencias de lenguaje, matemáticas y ciencias ciudadanas. Bogotá: Ministerio de Educación. Recuperado el 22 de Marzo de 2019, de https:/www.google.com/ search?rlz=1C1GGRV_enCO751CO751\&biw=1366\&bih=657\&ei=GMCVXKK6 Fu_m5gKbg7vAAQ\&q=Mnisterio+de+Educación

Eiras, M., Galván, C., Moragas, F., Obregon, C., Pisano, A., Romagnoli, A., \& Russel, R. (2018). Manual de lectura, escritura y oralidad académicas para ingresantes a la universidad. Quilmes, Argentina: Universidad Nacional de Quilmes. Recuperado el 23 de Marzo de 2019, de https://www.academia.edu/36135025/Manual_de_ lectura_escritura_y_oralidad_academicas_para_ingresantes_a_la_universidad

Pérez G. (1990). Investigacion-accion, aplicacion al campo educativo. Madrid: Dykinson. Recuperado el 21 de Marzo de 2019, de https:/www. casadellibro.com/libro-investigacionafaccion3a-aplicaciones-al-campo-socialeducativo/9788486133795/348261

Pérez, M. (2005). Evaluación de la comprensión lectora: dificultades y limitaciones. (C. y. Ministerio de Educación, Ed.) Revista de Educación, 121-138. Recuperado el 21 de Marzo de 2019, de http://www.revistaeducacion.mec.es/re2005.htm

Ramos, J., Cuetos, F., \& Arrivas, D. (2016). Evaluación de los procesos lectores en secundaria y babillerato PROLEC-SE-R. Mexico: TEA. Recuperado el 21 de mARZO de 2019, de https://www.casadellibro.com/libro-investigacionafaccion3a-aplicaciones-alcampo-social-educativo/9788486133795/348261

Rey, A. (2000). La enseñanza de la lectura en Colombia (1870-1930). Una aproximación desde el análisis del discurso. (C. d. Universidad Distrital Francisco José de Caldas, Ed.) Bogotá, Colombia: Grupo Editorial Gaía.

Sole, I. (Diciembre de 1992). Estrategias de lectura. Grao, 24-35. Recuperado el 22 de Marzo de 2019, de https://media.utp.edu.co/referencias-bibliograficas/uploads/ referencias/libro/1142-estrategias-de-lecturapdf-N0aU6-libro.pdf

Tobón, L. (2001). La lingüística del lenguajeeEstudios en torno a los procesos de significar y comunicar. aei. Recuperado el 21 de Marzo de 2019, de editorial. pedagogica.edu.co/verpub.php?pubid $=58$ 